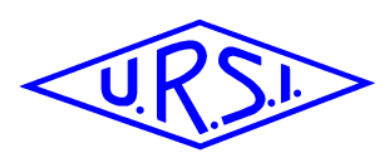

\title{
Inventory of Piled metal tubes using RFID technology.
}

\author{
Andrea Michel*(1), Marcos R. Pino ${ }^{(2)}$, Guillermo A. Narciandi ${ }^{(2)}$ and Paolo Nepa ${ }^{(1)}$ \\ (1) Dept. Information Engineering, University of Pisa, Italy [andrea.michel@unipi.it, paolo.nepa@unipi.it] \\ (2) Group of Signal Theory and Communications, Universidad de Oviedo, Spain [mpino@uniovi.es, \\ alvareznguillermo@uniovi.es]
}

\begin{abstract}
The performance in terms of reading range and read rate of passive RFID tags operating at UHF band is usually measured or calculated by assuming free-space propagation and local plane wave illumination conditions. In this paper, a practical scenario that does not meet the above assumptions has been analyzed. In particular, the RFID signal propagation and tag detection inside a guiding structure has been here considered. Theoretical considerations have been discussed to give practical guidelines for detecting a tagged metal pipe by means of a commercial UHF RFID reader.
\end{abstract}

\section{Introduction}

Radio Frequency Identification (RFID) systems have been largely used for logistic, retail and pharmaceutical applications and they are typically subdivided into two main groups on the basis of the operative frequency band: High Frequency (HF, $13.56 \mathrm{MHz})$ and Ultra High Frequency (UHF, 865-928 MHz) RFID bands [1]. HF RFID systems are employed for short-range applications (e.g. proximity identification and payments) and they are based on inductive coupling between coils. On the other hand, UHF RFID systems are used for both near-field (NF) and far-field (FF) applications such as localization, sensing and Item Level Tagging (ILT) applications [2-7]. UHFRFID systems have attracted increased attention because of the capability of detecting a higher number of tags with a higher reading rate.

The increasing number of RFID applications has given rise to practical issues which deserve specific attention and analysis. As an example, ad hoc antennas have been designed to be integrated inside the interrogator (also named as reader or encoder) case, which is typically compact in size or low profile. This is the case of antennas for desktop readers [3-5] or portable readers [6]. Moreover, in a number of ILT applications the tag detection must be confined within a limited volume close to the reader antenna, as for example in smart point readers [4] or Printer Encoders [7]. This is the case of the so-called Near-field UHF RFID applications, whose goal is to maximize the electric and magnetic field close to the antenna surface while limiting the far field radiation [3]. It is clear that the requirements for NF UHF-RFID antenna design significantly differ from those for conventional far-field focused antennas.

In this paper, a specific application in an unconventional operative scenario is taken into account, which is represented by the identification and inventory of metal pipes equipped with internal UHF RFID tags. For example, let us consider a number of tagged metal pipes piled in a storage area. By using a commercial handheld reader, a human operator or a drone is able to quickly inventory the items thanks to the UHF RFID technology. However, since the pipe is a metal structure that can be approximated with rectangular or circular waveguides, the RFID signal propagation inside the structure must be analyzed taking into account the modal propagation analysis. Hence, in this paper a preliminary propagation model is presented aiming at establishing a relation among reader output power, reader position, metal pipe size and tag position and orientation inside the pipe. In Section 2 the numerical and experimental analysis is described and discussed. A system-level test is also presented to qualitatively validate the proposed propagation model.

\section{Numerical and Experimental Analysis}

A metal pipe can be modeled from an electromagnetic (EM) point of view as a guided structure. Specifically, the EM propagation inside a cylindrical metal pipe such as an exhaust pipe can be modeled by a circular waveguide with radius $a$ and length $L$. At the ETSI UHF RFID band (865$868 \mathrm{MHz}$ ), the fundamental mode $\mathrm{TE}_{11}$ propagates when the radius $a$ is greater than $10 \mathrm{~cm}$. The analysis presented in this paper focuses on commercial pipes with $a=12.5 \mathrm{~cm}$ and $L=2 \mathrm{~m}$, where only the fundamental mode can propagate in the European UHF-RFID band.

As discussed in [8], the power received by a dipole-like tag placed inside the metal pipe depends on its position and orientation, and it is related to the electric field distribution of the propagating mode. Hence, the mode-to-tag coupling represents an important factor in the tag detection. In particular, the mode-to-tag coupling is higher at the center $(\mathrm{d}=0 \mathrm{~cm})$ of the waveguide where the $\mathrm{TE}_{11}$ E-field is 
oriented along the tag direction and the field level presents a maximum. As the tag is moved close to the external conductor, the mode-to-tag coupling decreases up to 5-6 $\mathrm{dB}$ since the $\mathrm{TE}_{11}$ E-field magnitude decreases and its orientation does not follow the dipole-like alignment.

This means that the position and orientation of the tag placed inside the metal pipe is a key factor to be considered to achieve satisfactory UHF RFID system performance. Furthermore, in an operative scenario, the UHF RFID reader is far from the metal pipe aperture, and thus the free space propagation losses as well as the coupling between the electric field radiated by the antenna and the propagating mode inside the waveguide must be included in the propagation model. Without lack of generality, in this analysis the portable reader is located at a distance $R$ from the metal pipe, at an angle $\theta$ with respect to the normal axis of the pipe, as schematically represented in Figure 1.

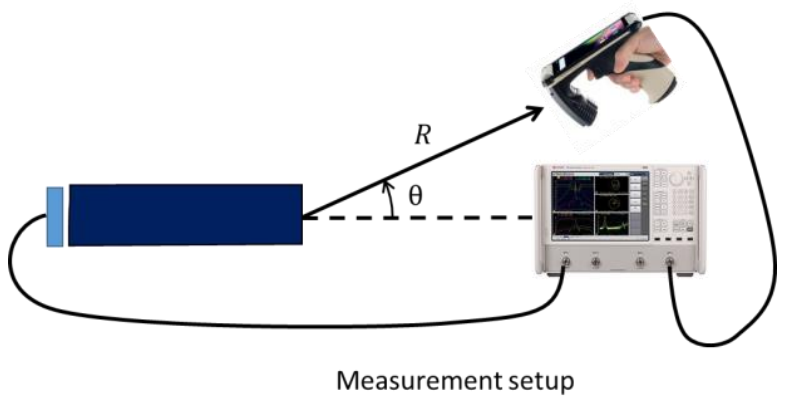

Figure 1. Measurement setup with both antennas connected to a VNA.

Initially, the coupling between the reader antenna and the propagating mode inside the waveguide has been measured by means of a 2-ports Vector Network Analyzer (VNA). A linearly polarized patch antenna has been placed close to a $2-\mathrm{m}$ long metal pipe with $a=12.5 \mathrm{~cm}$ and connected to the VNA. A second linearly polarized antenna has been placed at a distance $R$ from the other pipe open termination. Then, the $S_{12}$ parameter has been collected by varying the second antenna position, i.e. by varying $R$ and $\theta$. The measured $\mathrm{S}_{12}$ distribution at the central frequency of the ETSI UHF RFID band is plotted in Figure 2.

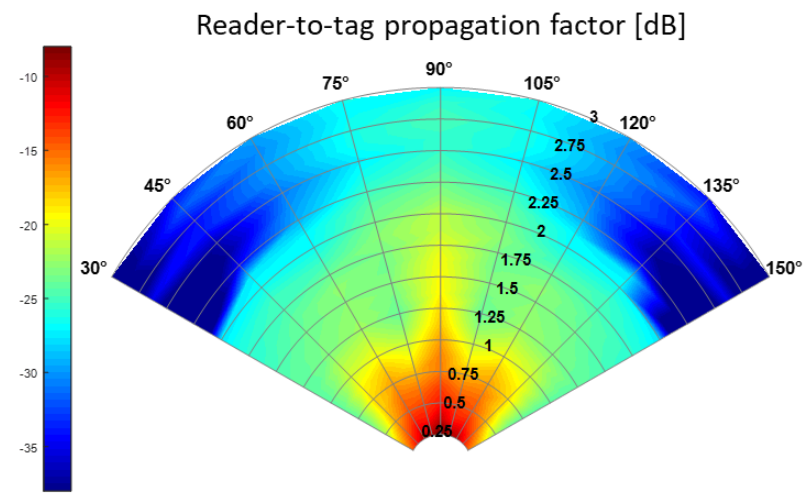

Figure $2 S_{21}$ parameter distribution measured with a VNA to model the RFID air-propagation and waveguide coupling.
An equivalent scenario has been modeled in the commercial software CST Microwave Studio ${ }^{\circledR}$. Specifically, by using Waveguide Ports at both the circular waveguide terminations, it is possible to estimate the coupling between the $\mathrm{n}^{\text {th }}$ propagating mode inside the waveguide and the dipole-like tag layout placed in the center of the waveguide $(\mathrm{d}=0 \mathrm{~cm})$. For the numerical and experimental analysis described in this paper, a dipole-like LAB ID UH100 Inlay tag has been taken into account. Due to the size of the circular waveguide, only the fundamental mode propagates, and all the other higher order modes are in cut-off. Finally, by adding the coupling between the field radiated by the reader antenna and the mode propagating inside the waveguide it is possible to estimate the power available at the waveguide section where the tag is placed on. Then, by also including the mode-to-tag coupling estimated by using CST Microwave Studio ${ }^{\circledR}$ it is possible to estimate the power received by the UHF RFID chip, modeled with a complex discrete port. It is worth noting that the received power can be estimated for each position of the reader antenna, creating the colormap show in Figure 3 . By considering a chip sensitivity equal to $-20 \mathrm{dBm}$, it is possible to estimate the region where the reader antenna should be placed in order to detect the tag inside the circular waveguide.

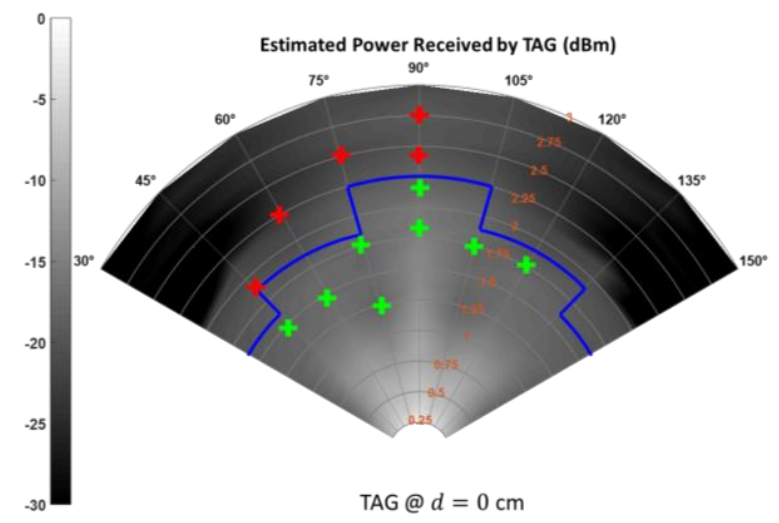

Figure 3. Estimated power received by the dipole-like LAB ID UH100 Inlay tag as a function of the position $(R, \theta)$ of the reader antenna. The blue line encloses the area where the estimated power received by the tag is over $-20 \mathrm{dBm}$. The green and red crosses indicate that the tag is detected or missed, respectively, when a commercial reader output power of $15 \mathrm{dBm}$ is set.

A system level test has been done to validate the results obtained by the afore-described analysis. Specifically, the linearly polarized patch antenna used to measure the $S_{12}$ parameter in Figure 2 has been connected to the Impinj Speedway R420 reader and fed with a power of $15 \mathrm{dBm}$. A LAB ID UH100 Inlay tag has been placed in the center of the circular waveguide. By varying the position and orientation of the antenna with respect to the metal pipe aperture, detection tests have been performed and summarized in Figure 3. The green crosses indicate the positions where the tag has been properly detected, while the red crosses indicate the positions where the tag detection is not possible. It should be observed that relatively high probability of tag detection is achieved 
when the reader antenna is located inside the region where a power higher of $-20 \mathrm{dBm}$ is estimated (enclosed in blue), confirming the effectiveness of the proposed propagation model.

\section{Acknowledgements}

This work has been supported by the Mobility Grants of the University of Oviedo financially supported by Banco de Santander and by the FPU grant of the Spanish Ministerio de Educación, Cultura y Deporte with reference FPU15/06431.

\section{References}

1. K. Finkenzeller, "RFID Handbook: Fundamentals and Applications in Contactless Smart Cards and Identification", 3rd ed. Hoboken, NJ: Wiley, 2010.

2. A. Buffi, M. R. Pino and P. Nepa, "Experimental Validation of a SAR-Based RFID Localization Technique Exploiting an Automated Handling System," IEEE Antennas and Wireless Propagation Letters, vol. 16, pp. 2795-2798, 2017, doi: 10.1109/LAWP.2017.2747216

3. A. Michel, P. Nepa, X. Qing and Z. N. Chen, "Considering High-Performance Near-Field Reader Antennas: Comparisons of Proposed Antenna Layouts for Ultrahigh-Frequency Near-Field Radio-Frequency Identification," IEEE Antennas and Propagation Magazine, vol. 60, no. 1, pp. 14-26, Feb. 2018, doi: 10.1109/MAP.2017.2774141

4. A. Michel, M. Rodriguez-Pino, and P. Nepa, "Reconfigurable modular antenna for near-field UHF RFID smart point readers," IEEE Trans. Antennas Propag., vol. 65, no. 2, pp. 1-9, Feb. 2017, doi: 10.1109/TAP.2016.2640140

5. C. Huang, C. Wang, J. Zhu and W. Tang, "A Reader Antenna for UHF Near-Field RFID Applications Based on the Segment-Line Oppositely Directed Currents," to appear on IEEE Antennas and Wireless Propagation Letters, doi: 10.1109/LAWP.2018.2867652

6. J. Zhang and Z. Shen, "Dual-Band Shared-Aperture UHF/UWB RFID Reader Antenna of Circular Polarization," IEEE Trans. Antennas Propag., vol. 66, no. 8, pp. 3886-3893, Aug. 2018, doi: 10.1109/TAP.2018.2839883

7 A. Buffi, A. Michel, P. Nepa, and G. Manara, "Numerical analysis of wireless power transfer in near-field UHFRFID systems" Wireless Power Transfer, pp. 1-12, 2017, doi:10.1017/wpt.2017.16

8. A. Michel, M. R. Pino and P. Nepa, "Detection of UHF RFID Tags in Metallic Guided Structures," 2018 2nd URSI Atlantic Radio Science Meeting (AT-RASC), Gran
Canaria, 2018, pp. 1-3, doi: 10.23919/URSI-ATRASC.2018.8471653 